\title{
BUFFER TYPES AND METHODS OF DEPLOYMENT IN CONSTRUCTION
}

\author{
Fernanda S. Bataglin ${ }^{1}$, Daniela D. Viana ${ }^{2}$, Rafael V. Coelho ${ }^{3}$, Iris D. Tommelein ${ }^{4}$, \\ and Carlos T. Formoso 5
}

\begin{abstract}
Flow is a key concept in Lean Production and is particularly important in construction. Due to the complexity of projects, in part due to managerial practices adopted, much variability exists in construction resource flows. Production system design can be used to eliminate at least some unwanted variability and then reduce the impact of remaining variability by using buffers in order to improve such flows. Accordingly, planners may add buffers of certain sizes in certain locations into the system, or use more systematic, adaptive, data-driven methods. With this in mind, the authors initiated a systematic literature review (SLR) on buffers in construction. The paper contributes to knowledge by defining the term 'buffer' and providing a characterization of buffer types and methods of deployment. Despite advances in understanding and method development, no one method stands out. The methods as described fall short of being able to both proactively determine buffer sizes and locations in the production system to suitably accommodate anticipated needs, and also reactively adjust them in light of system design changes. The use of SLR as the research methodology has well-known limitations, but the findings were revealing, and follow-on research will cast a wider net for relevant literature.
\end{abstract}

\section{KEYWORDS}

Buffer, slack, contingency, flow, variability.

\section{INTRODUCTION}

The concept of flow plays a key role in Lean Production, changing the way production traditionally is understood. Lean production systems are designed ideally to transform inputs into outputs while striving for instant delivery, minimizing resource use, as well as maximizing value, thus enabling customers to better accomplish their purposes (Ballard et al. 2001). Flow is particularly important in construction as construction pertains to complex, one-of-a-kind products and is undertaken usually at the delivery point by a series of repeating but variable activities in multiple locations within a multi-skilled adhoc team (Kenley 2005). This makes the Lean ideal particularly difficult to achieve.

1 PhD Candidate, Postgraduate Program in Civil Engineering: Construction and Infrastructure (PPGCI), Federal University of Rio Grande do Sul (UFRGS), Porto Alegre 90035-190, Brazil, fernanda.saidelles@gmail.com, orcid.org/0000-0001-8859-1201

2 Adjunct Professor, PPGCI, UFRGS, dietz.viana@ufrgs.br, orcid.org/0000-0001-8958-4708

3 Graduate Student, Civil and Envir. Eng. Dept., University of California, Berkeley, CA 94720-1712, USA, rvcoelho@ berkeley.edu, orcid.org/0000-0003-3298-3622

4 Professor, Civil and Envir. Eng. Dept., University of California, Berkeley, CA 94720-1712, USA, tommelein@ berkeley.edu, orcid.org/0000-0002-9941-6596

5 Professor, PPGCI, UFRGS, formoso@ufrgs.br, orcid.org/0000-0002-4772-3746 
Shingo (1989) defines production flow as a network of processes (flow of material or product in time and space) and operations (flow of workers and equipment in time and space). Ballard (2000) states that a requirement for good production flow is reliability, i.e., the necessary resources must be available at the right time to have a stable and predictable production system. The term resource refers to "a useful or valuable possession or quality that a person or organization has, for example, money, time, or skills" (Cambridge Dictionary 2021). In construction, resources are what is needed to execute a task, i.e., materials, information, workers, equipment and tools, space, time, and money.

Due to the peculiarities of construction projects and planning and control practices adopted, much variability exists in resource flows, and the probability of missing inputs is therefore considerable (Koskela 2000). Here, variability refers to "the quality of nonuniformity of a class of entities" (Hopp and Spearman 2011 p. 265). This can take on many forms, including process time variability and flow variability. Process variability stems from work procedure variations, setups, random outages, and quality problems. The related concept, process capability, refers to the characterization of such variability in the output of a process (or operation) under normal operating conditions and possibly adjusted for each project context by means of a probability distribution (Tommelein 2020). Hopp and Spearman further define that flow variability is created by the way work is released into the system or moved from one location to the next. Their key point is that the reduction of variability improves flow, yielding better results in production system performance. One way to reduce variability is to judiciously use buffers.

Production system design (PSD) is concerned with the development of operation and process design in alignment with product design, the structure of supply chains, and the allocation of resources (Ballard et al. 2001). It is an initial planning task that involves decisions that play an important role in the implementation of core Lean concepts, such as pull production, batch size, takt time (Schramm et al. 2006). PSD can be used to eliminate unwanted variability and reduce the negative impacts of the remaining variability by using buffers (Lee et al. 2006, Russell et al. 2015, Tommelein 2020).

So what are buffers and why use them? The term buffer is defined as a means to isolate operations subcycles from immediate interaction, i.e., to make them loosely linked (Howell et al. 1993). Buffers are resource cushions that can shield production from variability and thus help achieve desired outcomes (Alves and Tommelein 2004, Lee et al. 2006, Russell et al. 2015, Poshdar et al. 2018). Buffers make it possible to isolate a production process from its environment and also from the processes depending on it (González et al. 2011). Buffers are said to be redundancies that allow structural arrangement for the systems to accommodate variability (Miranda et al. 2007). However, in our view buffering does not mean that resources are necessarily redundant, e.g., standby capacity is related to the concept of underloading (i.e., intentionally scheduling resources to not be $100 \%$ utilized) in Takt Planning (TP) (Tommelein 2020) and helps to achieve reliability in systems subject to variability, i.e., most systems in the real world.

Terms related to buffer, are contingency and slack. The term contingency refers to a kind of buffer with time and money being the resources of concern. Contingency can cover possible time-cost-estimating errors and acts as a cushion against predictable as well as unforeseen risks (Barraza 2011).

The term slack generally appears to be given a broader meaning than buffer, with buffers being a kind of slack (Formoso et al. 2021, Saurin et al. 2021). Slack is more strategic in nature and of concern at the organizational level, whereas buffers tend to be more operational in nature and of concern at the project level. Slack is present when extra 
resources are available to allow an organization to adapt, change, and protect critical processes from internal pressures for adjustment or to external pressures for change in policy (Bourgeois 1981, Lawson 2002). One way of adapting is to use a buffer to insulate the organization's technical core from environmental changes (Moreno et al. 2009).

Buffers can serve as countermeasures to address production system performance concerns (Spear and Bowen 1999) and are then considered to be of value instead of waste. However, buffers that are not carefully sized and located can be wasteful and have negative impacts on the system, such as causing long lead times, inflating work-inprocess (WIP), and increasing non-value-adding activities (e.g., multiple handling of materials) (Howell and Ballard 1995). Likewise, oversized inventory buffers that are a consequence of the traditional idea that resources (workers and equipment) should be always kept busy (i.e., maximizing their utilization) even when the performed tasks are not immediately needed are also wasteful.

A common practice in construction is to add time buffers to a project schedule using a deterministic approach, not considering the dynamic nature of projects, e.g., Poshdar et al. (2018) mentioned the practice of simply adding a fixed percentage of the expected duration to each activity in a project network. This percentage may be decided according to the personal judgment and experience of project managers, "a trial-and-error process with dubious results" (González et al. 2011 p. 715). An alternative to using deterministic approaches is to use systematic, adaptive, data-driven methods, based on probabilistic mathematical models to define buffers and adjust them in real-time as needed.

The aim of our research is to categorize buffering methods used in construction as presented in the literature. We are not aware of literature that does this but think that a categorization can help in choosing which methods to use, alone or in combination, and under what circumstances. The research question addressed is: What buffer types of buffers and methods of deployment have been used for buffer management? This paper reports on the results of the first stage of this research, consisting of conducting an exploratory literature review. It is the start of an iterative process (Lavallee et al. 2014).

\section{RESEARCH METHODOLOGY}

The research is based on a systematic literature review (SLR), which is a means of identifying, evaluating, and interpreting documents relevant to a specific research question (Kitchenham 2004, Lavallee et al. 2014). The use of SLR as the methodology has well-known limitations, but the findings were nevertheless revealing, and follow-on research will cast a wider net for additional documents.

The SLR used four databases: (1) Science Direct, (2) American Society of Civil Engineers (ASCE), (3) Taylor \& Francis Online, and (4) International Group of Lean Construction (IGLC). The choice of these databases was admittedly somewhat arbitrary, but a starting point was needed for the review. The search used the terms \{ "buffer" OR "contingency" OR "slack" (in the title, abstract, and keywords) AND "planning" AND "control" AND "management" AND "construction project" (anywhere in the paper). The first three were chosen because they appear to be used interchangeably; the others were used to limit the scope of the search in this first stage of exploration.

A challenge in conducting this review is the use of certain terminology. Planning and control refer respectively to proactive (preventing anticipated disturbances as early as they are foreseeable) and reactive methods (incrementally repairing the plan in response to internal and external events that cause deviation from the plan, after Miranda et al. (2007), although planning is loosely used for both. Different authors use the term buffer 
with different meanings or fail to define the term altogether. This challenge was addressed during the review (see earlier definitions of buffer, etc.).

The authors accessed the databases between August 10 and 13, 2020, and identified 336 papers $^{6}$. Figure 1 illustrates the selection process steps. Use of these search criteria meant that certain papers were not found and consequently were not included in the review, although they might have been relevant (incl. several of our own papers that are relevant). Similarly, some papers found were judged to not suit the topic of interest and consequently were not included (e.g., papers in the domain of ecology).

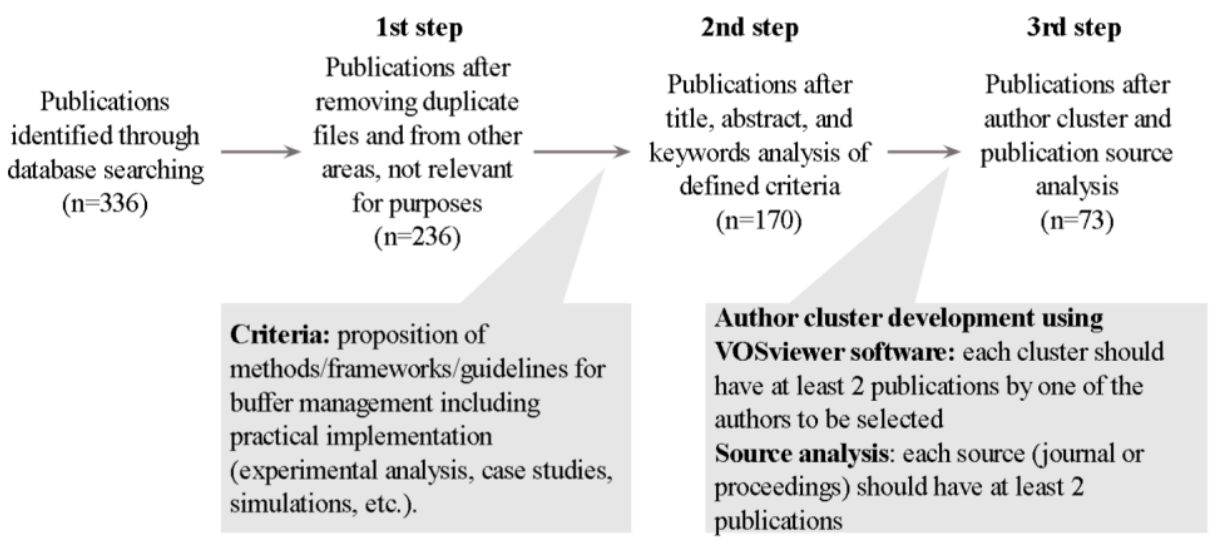

Figure 1: Steps of the systematic literature review

Assuming that authors (incl. teachers and students) may develop not one but several papers along a similar line of thought, we organized the papers by author cluster. The simultaneous review by cluster of multiple papers using related models and paradigms helped us gain better understanding of the authors' work. As a result of the overlapping between author clusters and source distribution analysis, 73 papers were selected for analysis. These formed 19 clusters (Figure 2). For brevity of this IGLC paper, limited to 10 pages, we report only the analysis of clusters with at least 3 papers and at least 3 authors per cluster (circled in Figure 2), which represent approximately $64 \%$ of all papers identified in the SLR.

Methods of buffer deployment were classified as proactive or reactive. They were also categorized according to different planning levels, considering the planning horizons adopted in the Last Planner® System (LPS) (Ballard and Tommelein 2021): (1) longterm: set milestones to be achieved during the execution of the project, (2) medium-term: identify and remove constraints by ensuring that necessary resources are made available, and (3) short-term: define and commit to work assignments that drive the production process.

\section{RESEARCH FINDINGS}

\section{BIBLIOMETRIC INFORMATION}

The 73 papers appeared in 11 different journals and conference proceedings with $75 \%$ of those being published over the last 15 years (since 2005), but some going as far back as 1994. The largest number of papers (44 in total) appeared in the Annual Conference of IGLC, and the next largest number (16) appeared in the ASCE Journal of Construction

\footnotetext{
${ }^{6}$ Readers may contact fernanda.saidelles@gmail.com to request the complete list of references.
} 
Engineering and Management. The paper's first authors were mostly from the United States (27 papers), Brazil (7), Chile (6), and New Zealand (6).

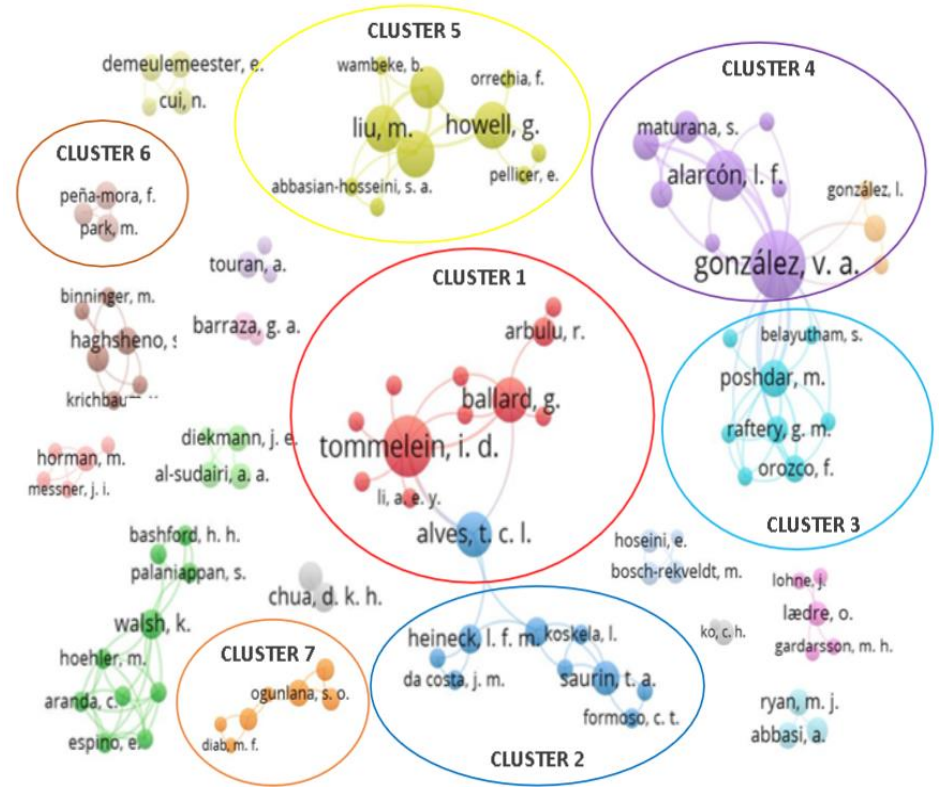

Figure 2: Network and clusters visualization

Authors from different clusters usually favored the use of one search term (either "buffer", "contingency", or "slack") over the other two, e.g., some authors who emphasized time and cost buffers used the term "contingency." Only the authors from Cluster 2 used the term "slack." They acknowledged that in Lean Construction the term "buffer" is more commonly used yet they preferred using "slack" because of its broader meaning. The IGLC papers by Formoso et al. (2021) and Saurin et al. (2021) discuss the concept of slack in greater depth and offer examples.

\section{BUfFERING METHODS OF DEPLOYMENT}

Buffering methods of deployment are identified based on the authors' cluster definition (numbers shown in Figure 3). Each of these is related to buffer types (space, capacity, information, time, inventory, and financial) based on the type of resource (space, workers, equipment/tools, material, information, time, and money). A sample of papers from these clusters is described next.

Frandson and Tommelein (2016) (Cluster 1) studied the use of TP for interior construction. The purpose is to create flow on a construction site based on a takt for each construction phase or process, i.e., each trade must complete their work in each assigned zone within the defined takt time. This method uses capacity buffers (underloading the resource) to balance the workflow. TP may be classified as a proactive method used for collaborative PSD, expanding on the commitment mechanism of the LPS.

Do et al. (2014) (Cluster 1) explored Target Value Design (TVD) as a method for controlling project cost overruns. Designing to a target cost is a product development practice that converts cost into a design criterion, rather than treat it as a design outcome. TVD projects, in contrast to other projects, require less contingency (financial buffer in this case) to cover a certain amount of uncertainty in the project because the project contingency gets pooled instead of being held individually by each participant. 


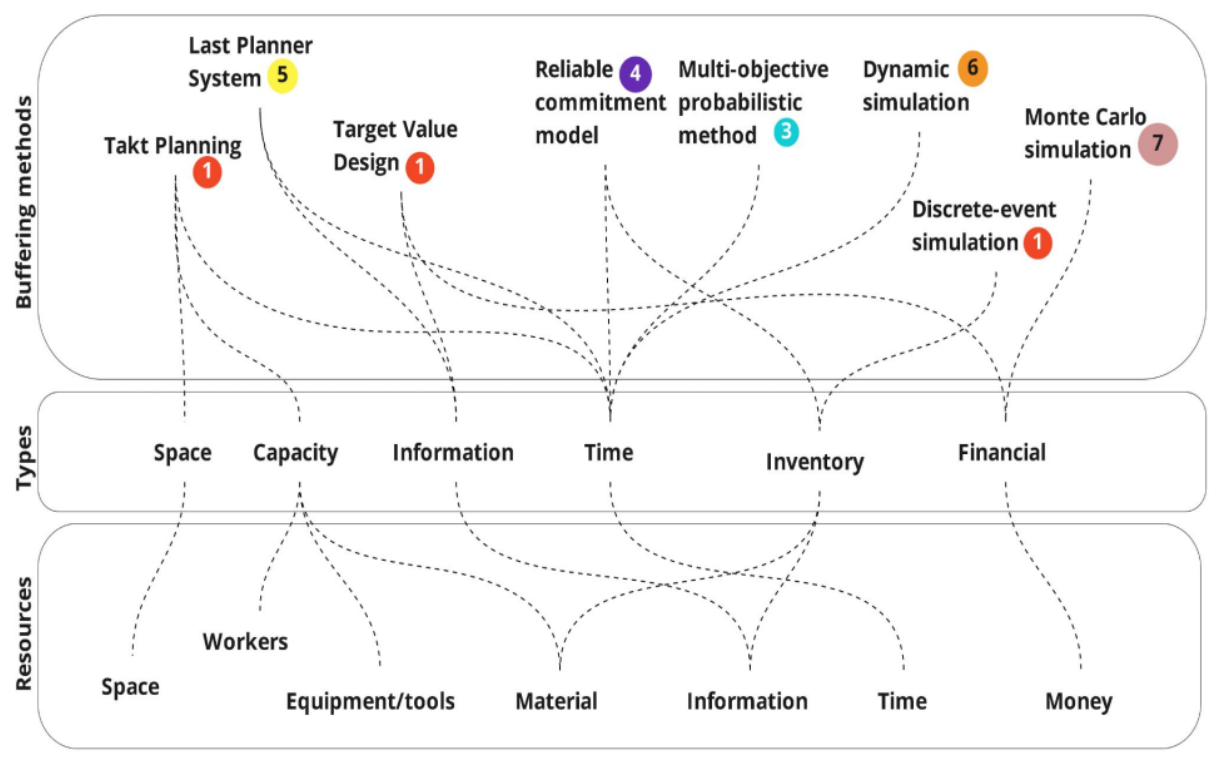

Figure 3: Buffering methods, types, and related resources

Alves and Tommelein (2004) (Cluster 1) used discrete-event simulation to mimic the behavior of the sheet-metal duct supply chain and how the choice of inventory buffers between activities impacts the system. The simulation revealed that if supply chain participants need large inventories between activities, lead times and WIP increase and the system's throughput decreases, a relationship described by Little's Law (Little 2011). Some contractors are sacrificing lead times and inventory levels for improvements in the reliability of their systems using buffers. The larger the buffer size, the longer it takes for the production batch to be assembled and released to the next activity, losing some advantages of the pull system. In this case, the supply chain loses its capacity to quickly respond to variations in demand. This amplifies the importance of first reducing the variation related to activities so that buffers can be reduced, and the system can be more effective. The simulation model offers a proactive approach because it allows understanding the system performance and prevents obstacles related to variability in this complex supply chain (incl. processes and operations), influencing all hierarchical levels of decision-making.

Poshdar et al. (2018) (Cluster 3) proposed using a multi-objective probabilistic-based time buffer allocation method (MPBAL) that was developed based on a mathematical solution to analyze construction project networks. They created models of variability at the activity level based on the information provided by project personnel and combined them into an integrated model that represents the probable time performance at the project level. Buffers are represented by the duration of the activities over their original expected duration. The project completion time, the project total cost, planning reliability, and schedule stability are the four criteria adopted to formulate the multi-objective problem of buffer allocation. MPBAL iteratively extends the duration of project activities by one unit of time buffer (e.g., 1 day) and quantifies their impact on the four criteria mentioned. The MPBAL method provided a range of options for the possible buffer allocation scenarios acting as a long-term proactive approach that gives decision-makers freedom to implement their preference in the final solution.

González et al. (2011) (Cluster 4) proposed a method for managing WIP buffers in repetitive projects, based on the reliable commitment model (RCM). RCM uses site information and planning reliability indicators that result in improved project 
performance, such as labor productivity and process progress. It provides a proactive approach for managing inventory buffers on construction projects at the operational level and demonstrates how WIP buffer size influences process capacity, resource use, and time delays. RCM aims to produce buffered work plans at the short-term level (weekly) using data from previous weeks to provide feedback into the system.

Russell et al. (2015) (Cluster 5) empirically demonstrated the effectiveness of the LPS in reducing time buffers and increasing Percent Plan Complete (PPC). Their findings demonstrate that the LPS exemplifies an effective planning strategy for construction managers to improve project performance and help them understand what drives the need for buffers in schedules, allowing efforts to strategically address areas of concern. Collection of time buffer, productivity, and PPC data demonstrated the effects of collaborative planning compared with traditional planning methods.

Lee et al. (2006) (Cluster 6) proposed a dynamic simulation-based buffering strategy to generate a construction plan, focusing on the detrimental impacts of errors and changes when concurrent design and construction are applied to a project. To absorb delays, this method places time buffers at the start of an activity, rather than at the end as a contingency buffer would. This time allows the performer of the activity to handle illdefined tasks by using a pre-checking or make-ready process to capture and correct predecessors' hidden errors (i.e., errors that have not been identified through the quality management process) and latent changes (i.e., changes that have not been identified through the scope management process nor approved through the claim and change management process). Thus, the method supports proactive behavior at the level of longand medium-term planning.

Panthi et al. (2009) (Cluster 7) focused on approaches to handle risks through the allocation of cost contingencies (financial buffers) on infrastructure projects such as highways, hydropower plants, and petroleum pipelines. Their method is divided into three steps: risk identification, risk assessment, and response. They quantified the effects of these risks by determining the probability and severity using the Analytical Hierarchy Process, and then in the response step, used Monte Carlo simulation to determine contingency. Theirs is a proactive approach because it was applied to allocate cost contingency for projects.

\section{DISCUSSION}

A variety of buffer types and methods of deployment were identified. Proactive methods are used during the planning process to anticipate possible problems and help to make decisions: methods focused on planning and control (Cluster 1); probabilistic and statistical models (Clusters 3 and 4); and tools such as simulations (Clusters 1, 6, and 7). Reactive methods use an outcomes analysis to determine a response based on how the results impacted the system performance (Cluster 5). Results from production system performance analysis, if reliable and consistent, may be used to generate data and provide feedback into the system, acting as inputs for proactive methods.

The scope for buffer allocation may be segmented into: (1) process (e.g., TP, LPS) vs. (2) operation (e.g., underloading with capacity), following Shingo's (1989) definition, and depending on the level of decision-making.

Based on the level of the planning system considered, buffer types can vary. Lee et al. (2006) (Cluster 6) added time buffers to the master schedule, whereas Alves and Tommelein (2004) (Cluster 1) added inventory buffers to the production schedule where details on resource allocation are visible. Also, some studies focused on a specific type 
of buffer, e.g., by Clusters 3 and 4 when probabilistic and statistical models are used. Others are more wide-ranging to allow decision-makers to choose what type of buffer they will prioritize, e.g., by Cluster 2 that focuses on design slack.

Saurin et al. (2013) (Cluster 2) illustrated the applicability of a set of guidelines for the management of complex socio-technical systems. One guideline suggested using design slack to reduce structural complexity and absorb the effects of unanticipated variability, which is a result of emerging events.

The concept of slack overlaps with the concept of buffer, but it has broader applicability. Cluster 2 authors recognized that in complex socio-technical systems, the concept of buffer is insufficient to deal with all possible types of variability. The evidence collected on a refurbishment project indicated that the master plan did not have any slack, regardless of its level of detail. According to the contractor's planner, a reason for no slack was the fact the client determined the handover dates, and there was little or no room for negotiating those dates. The planner also mentioned using, in some projects, a target plan that establishes a final handover date a few weeks before the date in the master plan. The target plan has best-case assumptions, and it creates a buffer to absorb delays that may yet happen. Whether or not the master plan has designed-in slack, it is worth noting that effects of unanticipated variability (e.g., schedule delays) can be dealt with not only by working longer hours and weekends but also by process and operation improvement (relying on human creativity) all of which are forms of slack capacity.

Fireman et al. (2018) (Cluster 2) investigated the role of slack in standardized work, assuming that it can be used to absorb variability from different sources. Standardized work is a type of action-oriented approach that sets a basis for continuous improvement and considers basic elements such as takt time, cycle time, WIP, and work sequence. Slack resources such as time, capacity, safety stocks, and also multifunctional team formation, cross-training, and the creation of help chains can enhance a project team's ability to deal with variability.

\section{CONCLUSIONS}

This paper provided an overview of buffering methods. The analysis of methods revealed that it appears to be impossible to define an absolute number for sizing buffers or to fix their location. The need for buffers is context-dependent and their use must be adapted to the nature of the system they pertain to. Construction projects are dynamic and buffering approaches must be able to adapt to changes in the system. A planner must understand the nature and functions in the system to decide where to invoke these functions' insights: in planning by adopting proactive methods or in reacting to circumstances. The deliberate use of buffers is important: if buffers are not well-managed they be wasteful instead of being of value by serving as a countermeasure to the manifestation of variability. Understanding sources of variability and removing unwanted variability must be done before adding buffers to reduce the impact of remaining variability in production systems.

Some studies proposed methods applicable to certain types of projects, nevertheless, for any type of project, the first step toward using buffers in construction management is to raise context awareness and answer questions such as: Are buffers needed, why?, What type of resources will be used as buffers?, and What type of methods of deployment will be used? Some methods are more complex to understand than others and consequently demand that users have prior knowledge of certain topics, e.g., mathematical modeling or simulation software. Such knowledge will affect which method users choose. 
We note that existing buffering methods can serve as alternatives to deterministic approaches traditionally used to define buffers in construction. Despite the advances in new method development (e.g., advances in mathematical modeling and simulation), further work is necessary to create more adaptive systems that allow for real-time decision-making and can respond when the need to use buffers arises. Having started with a SLR to come to explore existing literature on buffer management in construction, follow-on research will certainly broaden the search for related literature.

\section{ACKNOWLEDGMENTS}

This research was supported in part by the Coordenação de Aperfeiçoamento de Pessoal de Nível Superior - Brasil (CAPES) that sponsored the studies of Fernanda S. Bataglin at UC Berkeley and by members of the Project Production Systems Laboratory (P2SL). Any opinions, findings, conclusions, or recommendations expressed in this paper are those of the authors and do not necessarily reflect the views of CAPES or P2SL members.

\section{REFERENCES}

Alves, T. and Tommelein, I.D. 2004. "Simulation of buffering and batching practices in the interface detailing-fabrication-installation of HVAC ductwork." Proc. $12^{\text {th }}$ Ann. Conf. Int. Group for Lean Constr., Helsingør, Denmark, 1-13.

Ballard, G., Koskela, L., Howell, G., and Zabelle, T. 2001. "Production system design in construction" Proc. $9^{\text {th }}$ Ann. Conf. Int. Group for Lean Constr., Singapore, 1-15.

Ballard, G. and Tommelein, I.D. 2021. 2020 Benchmark of the Last Planner ${ }^{\circledR}$ System. Project Production Systems Laboratory, Univ. of California, Berkeley, CA, March.

Barraza, G. 2011. "Probabilistic estimation and allocation of project time contingency." J. Constr. Engin. Manage., 137(4), 259-265.

Bourgeois III, L.J. 1981. "On the measurement of organizational slack." Academy of Management Review, 6(1), 29-39.

Cambridge Dictionary 2021. https://dictionary.cambridge.org/us (accessed March 2021).

Do, D., Chen, C., Ballard, G., and Tommelein, I.D. 2014. "Target Value Design as a method for controlling project cost overruns." Proc. 22 $2^{\text {nd }}$ Ann. Conf. Int. Group for Lean Constr., Oslo, Norway, 171-181.

Fireman, M.C.T., Saurin, T.A., and Formoso, C.T. 2018. "The role of slack in standardized work in construction: an exploratory study." Proc. $26^{\text {th }}$ Ann. Conf. Int. Group for Lean Constr., Chennai, India, 1313-1322.

Formoso, C.T. et al. 2021. "Slack in construction - Part 1: Core concepts." Proc. $29^{\text {th }}$ Ann. Conf. Int. Group for Lean Constr., Lima, Peru, forthcoming.

Frandson, A. and Tommelein, I.D. 2016. "Takt time planning of interiors on a precast hospital project." Proc. $24^{\text {th }}$ Ann. Conf. Int. Group for Lean Constr., Boston, Mass., USA, 143-152.

González, V.A., Alarcón, L.F., Maturana, S., and Bustamante, J.A. 2011. "Site management of work-in-process buffers to enhance project performance using the reliable commitment model: case study." J. Constr. Engin. Manage., 137(9), 707-715.

Hopp, W. and Spearman, M. 2011. Factory Physics. $3^{\text {rd }}$ ed., Waveland Press, Illinois.

Howell, G. and Ballard, G. 1995. "Factors affecting project success in the piping function." Proc. $3^{\text {rd }}$ Workshop on Lean Construction, Albuquerque, USA, 161-185.

Howell, G., Laufer, A., and Ballard, G. 1993. "Interaction between subcycles: One key to improved methods." J. Constr. Eng. Manage., 119(4), 714-728. 
Kenley, R. 2005. "Dispelling the complexity myth: Founding lean construction on location-based planning." Proc. $13^{\text {th }}$ Ann. Conf. Int. Group for Lean Constr., Sydney, Australia, 245-251.

Kitchenham, B. 2004. Procedures for performing systematic reviews. Technical Report, Keele Univ., Keele, Staffs, UK, 28 pp.

Koskela, L. 2000. An exploration towards a production theory and its application to construction. VTT Technical Research Centre of Finland.

Lavallee, M., Robillard, P., and Mirsalari, R. 2014. "Performing systematic literature reviews with novices: An iterative approach." IEEE Trans. on Educ., 57(3) 175-181.

Lawson, M. 2002. "In praise of slack: time is of the essence." Academy of Management Perspectives, 15(3), 125-135.

Lee, S., Peña-Mora, F., and Park, M. 2006. "Reliability and stability buffering approach: focusing on the issues of errors and changes in concurrent design and construction projects." J. Constr. Engin. Manage., 132(5), 452-464.

Little, J. 2011. "Little's Law as viewed on its 50th anniversary." Operations Research, 59(3), 536-549.

Miranda, A.N., Costa, J.M., and Heineck, L. 2007. "Assessing the effects of structural differences on action, reaction and conformation in construction." Proc. $15^{\text {th }}$ Ann. Conf. Int. Group for Lean Constr., East Lansing, Mich., USA, 380-389.

Moreno, A, Fernandez, L., and Montes, F. 2009. "The moderating effect of slack resources on the relation between quality management and organisational learning." Int. J. of Prod. Research, 47(19), 5501-5523.

Panthi, K., Ahmed, S., and Ogunlana, S. 2009. "Contingency estimation for construction projects through risk analysis." Int. J. of Constr. Educ. and Research, 5(2), 79-94.

Poshdar, M., González, V.A., Raftery, G.M., Orozco, F., and Cabrera-Guerrero, G.G. 2018. "A multi-objective probabilistic-based method to determine optimum allocation of time buffer in construction schedules." Autom. in Constr., 92(1), 46-58.

Russell, M.M., Liu, M., Howell, G., and Hsiang, S.M. 2015. "Case studies of the allocation and reduction of time buffer through use of the Last Planner System." $J$. Constr. Engin. Manage., 141(2), 04014068.

Saurin, T., Rooke, J., Koskela, L., and Kemmer, S. 2013. "Guidelines for the management of complex socio-technical systems: An exploratory study of a refurbishment project." Proc. $21^{\text {st }}$ Ann. Conf. Int. Group for Lean Constr., Fortaleza, Brazil, 915-924.

Saurin, T.A. et al. (2021). "Slack in construction - Part 2: Practical applications." Proc. $29^{\text {th }}$ Ann. Conf. Int. Group for Lean Constr., Lima, Peru, forthcoming.

Schramm, F K., Rodrigues, A.A. and Formoso, C.T. 2006. "The role of production system design in the management of complex projects." Proc. 14 ${ }^{\text {th }}$ Ann. Conf. Int. Group for Lean Constr., Santiago, Chile, 227-239.

Shingo, S. 1989. A study of the Toyota production system from an industrial engineering viewpoint. Cambridge, Mass.: Productivity Press.

Spear, S. and Bowen, H. 1999. "Decoding the DNA of the Toyota Production System." Harvard Business Review, 77(5), 96-106.

Tommelein, I.D. 2020. "Takting the Parade of Trades: Use of capacity buffers to gain workflow reliability." Proc. $28^{\text {th }}$ Ann. Conf. Int. Group for Lean Constr., Berkeley, Calif., USA, 421-432. 